\title{
Low Glucose at 3-Hour 100 Gram Oral Glucose Tolerance Test: Implications for Glucose Control in Gestational Diabetes
}

\author{
Lee Reicher ${ }^{1}$, Anat Lavie $^{2}$, Guy Kern², Emmanuel Attali², larissa finmeser ${ }^{2}$, Isca \\ Landesberg $^{2}$, Relly Reicher ${ }^{3}$, Yariv Yogev ${ }^{3}$, and sharon maslovitz ${ }^{2}$ \\ ${ }^{1}$ Affiliation not available \\ ${ }^{2}$ Tel-Aviv Sourasky Medical Center, Tel-Aviv, Israel. Affiliated to the Sackler Faculty of \\ Medicine, Tel-Aviv University, Tel-Aviv, Israel. \\ ${ }^{3}$ Tel Aviv Sourasky Medical Center
}

October 14, 2020

\begin{abstract}
Objective: To assess the clinical significance of a low 180-minute glucose value in a 100gr oral glucose tolerance test (OGTT) and a single high abnormal value. Design: A retrospective cohort study. Setting: A single outpatient health clinic. Population: Women with one abnormal high OGTT glucose value. The study group included women with 180-minute plasma glucose levels of [?]60 mg/dl and one abnormal value in the OGTT. The control group was comprised of women with one abnormal value in the OGTT and normal 180-minute glucose value. Methods: Pregnancy related outcomes and level of glycemic control of both groups were compared. Main outcome measures: The primary outcome was glycemic control, defined as fasting blood glucose measurements $>90 \mathrm{mg} / \mathrm{dl}$ or post-prandial glucose values $>140 \mathrm{mg} / \mathrm{dl}$ or $120 \mathrm{md} / \mathrm{dl}$ (1-hour and 2-hour post-prandial, respectively) in $>30 \%$ of the measurements. Secondary outcomes were the rate of insulin treatment and the perinatal outcome consisting of birthweight, large-for-gestational-age and polyhydramnios. Results: 301 women were included, of them, 143 in the study group and 158 in the control group. Pre-pregnancy body mass index, first trimester fasting glucose levels, previous gestational diabetes mellitus, and familial diabetes were similar for both groups. Suboptimal glycemic control was more prevalent among the women in the study group ( $14 \%$ vs. $5.1 \%$, respectively, $\mathrm{P}=0.01)$. The need for insulin treatment was similar in both groups $(9.8 \%$ vs. $4.4 \%, \mathrm{P}=.1)$. Conclusion: Women with one abnormal value and a 180-minute hypoglycemia in the OGTT are at increased risk for suboptimal glycemic control.
\end{abstract}

\section{Introduction}

Gestational diabetes mellitus (GDM) developed into a worldwide major concern due to its increasing prevalence and clinical implications ${ }^{1}$. A variety of adverse perinatal outcomes, including macrosomia, shoulder dystocia, neonatal respiratory and metabolic disorders, and cesarean deliveries ${ }^{2}$ are associated with high maternal blood glucose levels. The long-term detrimental effects of GDM on metabolic function in adulthood have also been investigated and established. ${ }^{3}$

Detection of GDM is generally based on a two-step approach. The first step (glucose challenge test, GCT) is utilized to screen pregnant women and detect those at high risk for GDM by having the subjects consume $50 \mathrm{~g}$ of glucose and sampling their blood for glucose 1 hour later. Women whose glucose levels meet or exceed the screening threshold are then subjected to a 3-hour diagnostic oral glucose tolerance test (OGTT) consisting of a total of $100 \mathrm{~g}^{4}$. Whether one or two abnormally high values in the OGTT results define GDM is still under debate ${ }^{5}$. While some suggest that a single abnormal value is associated with increased risk of large-for-gestational age (LGA) infants ${ }^{6}$, cesarean delivery, pre-eclampsia ${ }^{7}$ and increased risk of Type- 2 diabetes later in life, others dispute these associations. ${ }^{8,910,11}$. A recent systematic review concluded that 
hyperglycemia-related adverse perinatal outcomes are similarly found among women with both one or two abnormal OGTT values ${ }^{12}$.

The finding of hypoglycemia three hours after consuming $100 \mathrm{~g}$ of glucose is not uncommon and may present with dizziness, nausea, tachycardia, and sweating ${ }^{13}$. Clinical experience led us to the impression that women with one abnormal high value in the OGTT and hypoglycemia in the 180-min value have a more pronounced insulin resistance. Our search of the literature for studies assessing the clinical implications of such hypoglycemia on glucose control and perinatal outcome among women with a single abnormally high value yielded no results. In light of the unassessed clinical significance of such a common occurrence, we were motivated to investigate this clinical quandary. We hypothesized that a 3-hour hypoglycemia finding may reflect impaired insulin response and may predict suboptimal glycemic control as evidenced on daily glucose profiles of pregnant women with a single abnormally high OGTT value.

\section{Methods}

We conducted a retrospective cohort study at a single outpatient health clinic between 2011 and 2018. The study group included healthy women at 24-28 weeks of gestation with one abnormally high value on their OGTT and a low glucose finding for the 3-hour hypoglycemia analysis $(<60 \mathrm{mg} / \mathrm{dl})$. The control group was comprised of women with high or normal 180-minute glucose values with one abnormal value in the OGTT. Excluded from the study were women with a multiple pregnancy, pre-gestational diabetes, or incomplete medical or obstetrical records. The Institutional Review Board approved the study and waived informed consent.

GDM was diagnosed by a two-step approach consisting of a 1-hour 50 g GCT at 24-28 weeks of pregnancy, followed by a 3-hour $100 \mathrm{~g}$ diagnostic OGTT if the GCT plasma glucose result was [?]140 mg/dL. Women with risk factors for developing GDM, such as a family history of diabetes in a first-degree relative, previous GDM, or previous macrosomia, underwent only diagnostic OGTT. GDM was diagnosed when at least one of the four OGTT glucose values was abnormally high by the Carpenter and Cousten criteria ${ }^{14}$.

Once diagnosed, all women were instructed to self-measure capillary glucose at least four times daily (fasting and one or two hours postprandial). The initial clinic visit included counseling by a nurse expert in diabetes, dietary consultation by a certified dietician, and consultation with a maternal-fetal medicine specialist. The biweekly visits were scheduled up to 36 weeks of gestation and weekly from that stage to delivery. Each visit included dietary consultation, blood pressure monitoring, urine dipstick test, review of daily glucose profiles, and decision-making regarding the need for adjusting therapy. Ultrasound scans for biophysical profiles and estimation of fetal growth were performed biweekly from 26 weeks of gestation, and non-stress testing was added at 34 weeks of gestation.

The desired level of glycemic control was defined as mean fasting blood glucose $<90 \mathrm{mg} / \mathrm{dl}$ and mean one or two hours postprandial $<140 \mathrm{mg} / \mathrm{dL}$ or $<120 \mathrm{mg} / \mathrm{dl}$, respectively. Women with fewer than 50 glucose measurements over the two weeks were not included in the final analysis. Women who had more than $30 \%$ abnormally high values during a 2 -week period of glucose monitoring were considered as having suboptimal glucose control. ${ }^{15}$ The detection of suboptimal glucose control led to an intervention aimed at modifying the diet and encouraging physical activity. Insulin treatment was initiated when fasting glucose levels were consistently $>100 \mathrm{mg}$ after two weeks of observing a strict diet or when suboptimal glucose control, as defined above, persisted after 2 weeks of dietary modifications and physical activity.

The need for induction of labor was determined by the level of glycemic control and estimated fetal weight. Induction of labor was offered at 38-39 weeks of gestation if the estimated fetal weight (EFW) was 3600-4000 gr or poor glycemic control was documented [defined as?]. Cesarean section was advised when the EFW was $>4000 \mathrm{gr}$ or for obstetrical indications. If the abovementioned criteria were not fulfilled induction of labor was advocated at 41 weeks of gestation.

Women with one abnormally high OGTT value were divided into two groups. Women in the study group had a low 180-minute value defined as a plasma glucose level of [?]60 mg/dl, and those in the control group had 
a 180-minute glucose value of $>60 \mathrm{mg} / \mathrm{dl}$. The following data were extracted from women's computerized files and compared between the two groups: maternal demographics and clinical characteristics, periods of suboptimal glucose control, the need for insulin to achieve desired levels of glycemic control, glycemic profile, and obstetrical and neonatal outcome measures. The primary outcome was the rate of women with suboptimal maternal glycemic control. The secondary outcomes were the need for insulin treatment to achieve desired levels of glycemic control and the presence of obstetrical and neonatal complications, including mean birthweights, incidence of macrosomia (birthweight more than the $90^{\text {th }}$ percentile or $>4000$ gr), and polyhydramnios (amniotic fluid index $>25 \mathrm{~cm}$ or a maximal vertical pocket $>8 \mathrm{~cm}$ ).

Statistical analysis

Between-group comparisons of the study participants' characteristics were performed by a 2-tailed Student's $\mathrm{t}$ test for continuous variables, a Pearson $\chi^{2}$ test for categorical variables, and the Kruskal-Wallis test for continuous non-normal variables. A $P$ value of less than or equal to .05 was considered significant. Multivariate logistic regression assessed the association between the various selected variables and suboptimal maternal glycemic control. Maternal age, parity, EFW, and delivery week were taken as continuous variables and the remaining variables were taken as categorical. All statistical analyses were performed with $\mathrm{R}$ version 3.5.2. (http://www.r-project.org)

\section{RESULTS}

Overall, 301 women were enrolled, of them, 143 women in the study group and 158 in the control group. The groups were similar with regard to demographic and clinical characteristics, including age, parity, and pre-pregnancy body mass index (BMI) (Table 1). Both groups were similar in the parameters considered as risk factors for insulin resistance and GDM, such as gestational weight gain, previous GDM, diabetes among first-degree relatives, glucose values during the first trimester, and GCT result.

The rate of suboptimal glucose control was higher in the study group ( $14 \%$ vs. $5.1 \%$ in comparison to the control group, $P=.01$ ) (Table 2). There was no significant group difference in the need for insulin treatment to achieve desired levels of glycemic control after dietary adjustment and enhanced physical activity. Other secondary outcome measures, such as birthweight, mode of delivery, and prevalence of hyperglycemia-related complications (polyhydramnios, macrosomia) were also similar between the study groups (Table 2).

A multiple logistic regression analysis showed that a low glucose value of 100 gr at 3 hours was associated with a higher incidence of suboptimal glucose control (odds ratio 3.1, confidence interval $[\mathrm{CI}]$ 1.35-7.83, $P$ $=.01$ ). The effects of factors that are traditionally considered predictors of insulin resistance on suboptimal daily glucose profile, such as BMI, first trimester fasting glucose, and weight gain, did not reach a level of significance (Fig. 1).

\section{Discussion}

The current study assessed the clinical implications of a low glucose 180 minutes value at the 3-hour OGTT among women with a single abnormally high OGTT value. It emerged that a suboptimal daily glucose profile was more prevalent among the women in the study group compared to women in the control group.

Insulin sensitivity has been shown to decline during pregnancy, triggering an enhanced insulin release that culminates in euglycemia after consumption of $100 \mathrm{gr}$ glucose during an OGTT ${ }^{16}$. Kuhl et al. reported that women with GDM have impaired first-phase insulin response which may result in late occurrence of peak plasma insulin concentrations during an OGTT ${ }^{17}$. Women with overly impaired insulin response may experience a delayed peak in insulin blood levels at 3 hours after glucose consumption and consequent late hypoglycemia. Thus, hypoglycemia at 3 hours may represent exceedingly impaired glucose tolerance.

Delibas et al. found an association between reactive hypoglycemia during an otherwise normal OGTT and adverse perinatal outcome measures, such as low Apgar scores, low birth weights, and prenatal admission to the neonatal intensive care unit. ${ }^{18}$ Since only women with a normal OGTT were included, the daily glucose profile was not assessed. Moreover, the sample size of their study group (pregnant women with 
reactive hypoglycemia) was relatively low. We were able to demonstrate a possible association between late hypoglycemia and compromised glucose control since our study group included women with a daily glucose profile that was investigated due to a single abnormal value.

To the best of our knowledge, this is the first study to describe a possible association between a low glucose result of a 3-hour $100 \mathrm{~g}$ diagnostic OGTT and maternal suboptimal glucose control. Daily glucose control is affected by multiple factors that are related to insulin resistance, such as fasting glucose levels during the first trimester, BMI, gestational weight gain, and previous GDM. We carried out a multivariate logistic regression analysis in order to assess the effect of late hypoglycemia evidenced during an OGTT on the glucose profile. As shown in Fig. 1, hypoglycemia at 3 hours was the only parameter associated with suboptimal glucose control with an odds ratio of 3.1 (CI, 1.35-7.83). Earlier studies had found associations between first or second trimester fasting glucose levels and the need for pharmacological intervention to achieve euglycemia in women with gestational diabetes ${ }^{19,20}$. In contrast to those findings, our study assessed women with a single abnormal value, under strict diet with similar first trimester fasting glucose values in both study and control groups. Moreover, as mentioned above, fasting glucose was not associated with suboptimal glucose control.

We anticipated that suboptimal glucose values would be reflected in the number of women requiring pharmacological treatment to lower their blood glucose levels, but the difference between the study and control groups failed to reach a level of significance. About $10 \%$ of the women in the study group needed insulin compared to $4.4 \%$ of the control group $(P=.1)$. This may be explained by efficient interventions, such as diet modification and enhanced physical activity, that allowed us to spare many women with suboptimal glucose control from the need for treatment. Our study has some limitations that bear mention. First, its retrospective nature makes it susceptible to selection bias, potential recording errors, and difficulty in controlling for exposures and outcomes. This limitation precluded us from arriving at any definitive conclusion about the impact of late hypoglycemia during the OGTT on perinatal parameters, we found no significant difference between the groups in neonatal outcomes, including Apgar scores at 5 and 10 minutes and prevalence of NICU admission. Additionally, the study was performed in a single center that treats a relatively homogenous population of characteristically young and multiparous women, thus generalization of the findings may be open to question. Finally, the relatively low numbers of participants prevented us from demonstrating the effect of late hypoglycemia on the need for pharmacological treatment.

This study revealed a significant association between hypoglycemia at 3 hours after glucose consumption during an OGTT and suboptimal maternal glycemic control among women with a single abnormally high value in their OGTT. Stringent follow-up and meticulous glucose control may be essential for this subgroup of women.

The authors report no conflict of interest.

Contribution to authorship:

Lee Reicher, Anat Lavie, Larissa Feinmesser - Manuscript writing/editing

Sharon Maslovitz, Yariv Yogev, Lee Reicher, - Protocol/project development

Emmanuel Attali, Relly Reicher, Isca Landesberg - Data collection and management

Guy Kern -Data analysis

Details of ethics approval: IRB TLV 0273-19 Jan 2019

This research received no specific grant from any funding agency in the public, commercial, or not-for-profit sectors

\section{REFERENCES}

1. Gabbe SG, Niebyl JR, Galan HL, et al. Obstetrics: Normal and Problem Pregnancies E-Book. Elsevier Health Sciences; 2016. 
2. Hartling L, Dryden DM, Guthrie A, Muise M, Vandermeer B, Donovan L. Benefits and harms of treating gestational diabetes mellitus: a systematic review and meta-analysis for the U.S. Preventive Services Task Force and the National Institutes of Health Office of Medical Applications of Research. Ann Intern Med . 2013;159(2):123-129. doi:10.7326/0003-4819-159-2-201307160-00661

3. Reece EA, Homko CJ. Infant of the diabetic mother. Semin Perinatol . 1994;18(5):459-469.

4. CoNN JW. Interpretation of the glucose tolerance test. The necessity of a stand ard preparatory diet. American Journal of Medical Sciences . 1940;199:555-564.

5. Committee on Practice Bulletins - Obstetrics. ACOG Practice Bulletin No. 190: Gestational Diabetes Mellitus. Obstet Gynecol . 2018;131(2):e49-e64. doi:10.1097/AOG.0000000000002501

6. McLaughlin GB, Cheng YW, Caughey AB. Women with one elevated 3-hour glucose tolerance test value: are they at risk for adverse perinatal outcomes? Am J Obstet Gynecol . 2006;194(5):e16-19. doi:10.1016/j.ajog.2006.01.028

7. Carr DB, Newton KM, Utzschneider KM, et al. Gestational diabetes or lesser degrees of glucose intolerance and risk of preeclampsia.Hypertens Pregnancy . 2011;30(2):153-163. doi:10.3109/10641950903115012

8. Kaufmann RC, McBride P, Amankwah KS, Huffman DG. The effect of minor degrees of glucose intolerance on the incidence of neonatal macrosomia. Obstet Gynecol . 1992;80(1):97-101.

9. Forest JC, Massé J, Garrido-Russo M. Glucose tolerance test during pregnancy: the significance of one abnormal value. Clin Biochem . 1994;27(4):299-304. doi:10.1016/0009-9120(94)00029-8

10. Fassett MJ, Dhillon SHK, Williams TR. Effects on perinatal outcome of treating women with 1 elevated glucose tolerance test value. Am J Obstet Gynecol . 2007;196(6):597.e1-4; discussion 597.e4. doi:10.1016/j.ajog.2007.03.017

11. Gruendhammer M, Brezinka C, Lechleitner M. The number of abnormal plasma glucose values in the oral glucose tolerance test and the feto-maternal outcome of pregnancy. Eur J Obstet Gynecol Reprod Biol . 2003;108(2):131-136. doi:10.1016/s0301-2115(02)00370-6

12. Roeckner JT, Sanchez-Ramos L, Jijon-Knupp R, Kaunitz AM. Single abnormal value on 3-hour oral glucose tolerance test during pregnancy is associated with adverse maternal and neonatal outcomes: a systematic review and metaanalysis. Am J Obstet Gynecol . 2016;215(3):287-297. doi:10.1016/j.ajog.2016.04.040

13. Weissman A, Solt I, Zloczower M, Jakobi P. Hypoglycemia during the 100-g oral glucose tolerance test: incidence and perinatal significance.Obstet Gynecol . 2005;105(6):1424-1428. doi:10.1097/01.AOG.0000159577.28448.f9

14. Carpenter MW, Coustan DR. Criteria for screening tests for gestational diabetes. Am J Obstet Gynecol . 1982;144(7):768-773. doi:10.1016/0002-9378(82)90349-0

15. American Diabetes Association. Standards of medical care in diabetes-2011. Diabetes Care . 2011;34 Suppl 1:S11-61. doi:10.2337/dc11-S011

16. Catalano PM, Tyzbir ED, Roman NM, Amini SB, Sims EA. Longitudinal changes in insulin release and insulin resistance in nonobese pregnant women. Am J Obstet Gynecol . 1991;165(6 Pt 1):1667-1672. doi:10.1016/0002-9378(91)90012-g

17. Kühl C. Etiology and pathogenesis of gestational diabetes.Diabetes Care . 1998;21 Suppl 2:B19-26.

18. Delibas IB, Tanriverdi S, Cakmak B. Does reactive hypoglycemia during the $100 \mathrm{~g}$ oral glucose tolerance test adversely affect perinatal outcomes? Ginekol Pol . 2018;89(1):25-29. doi:10.5603/GP.a2018.0005

19. Akinci B, Celtik A, Yener S, Yesil S. Is fasting glucose level during oral glucose tolerance test an indicator of the insulin need in gestational diabetes? Diabetes Res Clin Pract . 2008;82(2):219-225. doi:10.1016/j.diabres.2008.07.023 
20. Wong VW, Jalaludin B. Gestational diabetes mellitus: who requires insulin therapy? Aust $N$ Z J Obstet Gynaecol . 2011;51(5):432-436. doi:10.1111/j.1479-828X.2011.01329.x

Table 1. Demographic, Clinical and Obstetrical Characteristics

$\mathrm{P}$

Study group

$(\mathrm{n}=143)$

Control group

$(\mathrm{n}=158)$

\section{Characteristic}

0.52

$28.3 \pm 5.7$

$28.7 \pm 5.4$

Maternal age (years)

0.26

$47(32.9 \%)$

$44(27.8 \%)$

Nulliparous

0.59

$23.3 \pm 1.8$

$23.5 \pm 1.7$

Pre-pregnancy BMI $\left(\mathrm{kg} / \mathrm{m}^{2}\right)$

0.88

$28.35 \pm 2.1$

$28.3 \pm 2$

BMI at OGTT $\left(\mathrm{kg} / \mathrm{m}^{2}\right)$

0.38

$12.1 \pm 3.9$

$11.7 \pm 3.6$

Gestational weight gain $(\mathrm{kg})$

0.8

$22(15.4 \%)$

$27(17.1 \%)$

Diabetes mellitus in the family ${ }^{\mathrm{a}}$

1

$33(23.1 \%)$ 
$37(23.4 \%)$

GDM in previous pregnancies

0.26

$154.6 \pm 17.6$

$152.7 \pm 18.2$

Glucose challenge test $(\mathrm{mg} / \mathrm{dl})$

0.18

$86.7 \pm 7.5$

$87.9 \pm 8.2$

Fasting glucose in $1^{\text {st }}$ trimester $(\mathrm{mg} / \mathrm{dl})$

Data are presented as mean \pm standard deviation or $\mathrm{n}(\%)$

OGTT, oral glucose challenge test; BMI, body mass index; GDM, gestational diabetes mellitus.

${ }^{\text {a }}$ Defined as diabetes mellitus in a first-degree relative

Table 2. Primary, Secondary, and Obstetrical Outcome Measures

\begin{tabular}{|c|c|c|c|}
\hline $\mathbf{P}$ & $\begin{array}{l}\text { Study group }(n= \\
143)\end{array}$ & $\begin{array}{l}\text { Control group }(\mathrm{n}= \\
158)\end{array}$ & Characteristic \\
\hline & & & $\begin{array}{l}\text { Primary outcome } \\
\text { Women with } \\
\text { suboptimal glucose } \\
\text { control }^{\mathrm{a}}\end{array}$ \\
\hline 0.01 & $20(14 \%)$ & $8(5.1 \%)$ & Secondary outcome \\
\hline 0.1 & $14(9.8 \%)$ & $7(4.4 \%)$ & $\begin{array}{l}\text { Women needing insulin } \\
\text { treatment }^{\mathrm{b}}\end{array}$ \\
\hline 0.18 & $3138 \pm 807$ & $3005 \pm 900$ & Birthweight (grams) \\
\hline 0.63 & $14(9.8 \%)$ & $12(7.6 \%)$ & $\begin{array}{l}\text { Birthweight }>90^{\text {th }} \\
\text { percentile }\end{array}$ \\
\hline 0.18 & $15(10.5 \%)$ & $9(5.7 \%)$ & Polyhydramnios \\
\hline 1 & $8(5.6 \%)$ & $8(5.1 \%)$ & Preeclampsia \\
\hline 0.64 & $9(6.3 \%)$ & $7(4.4 \%)$ & 5 minutes Apgar $<7$ \\
\hline 0.46 & $39.2 \pm 1.2$ & $39.1 \pm 1.3$ & $\begin{array}{l}\text { Gestational age at } \\
\text { delivery (weeks) }\end{array}$ \\
\hline 1 & $22(15.4 \%)$ & $24(15.2 \%)$ & Cesarean delivery \\
\hline
\end{tabular}

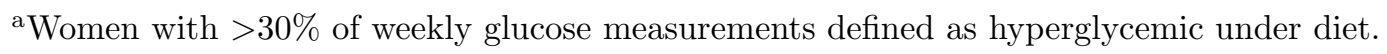

${ }^{\mathrm{b}}$ Number of women needing insulin to achieve desired glucose control after diet modification and physical activity have failed to do so.

Data are presented as mean \pm standard deviation or $\mathrm{n}(\%)$

OGTT, oral glucose challenge test; LGA, large-for-gestational age ( $>90 \%$ of population-based birth weight).

Fig. 1. Logistic regression plot of odds ratios and $95 \%$ confidence intervals for variables predicting suboptimal glucose control. 


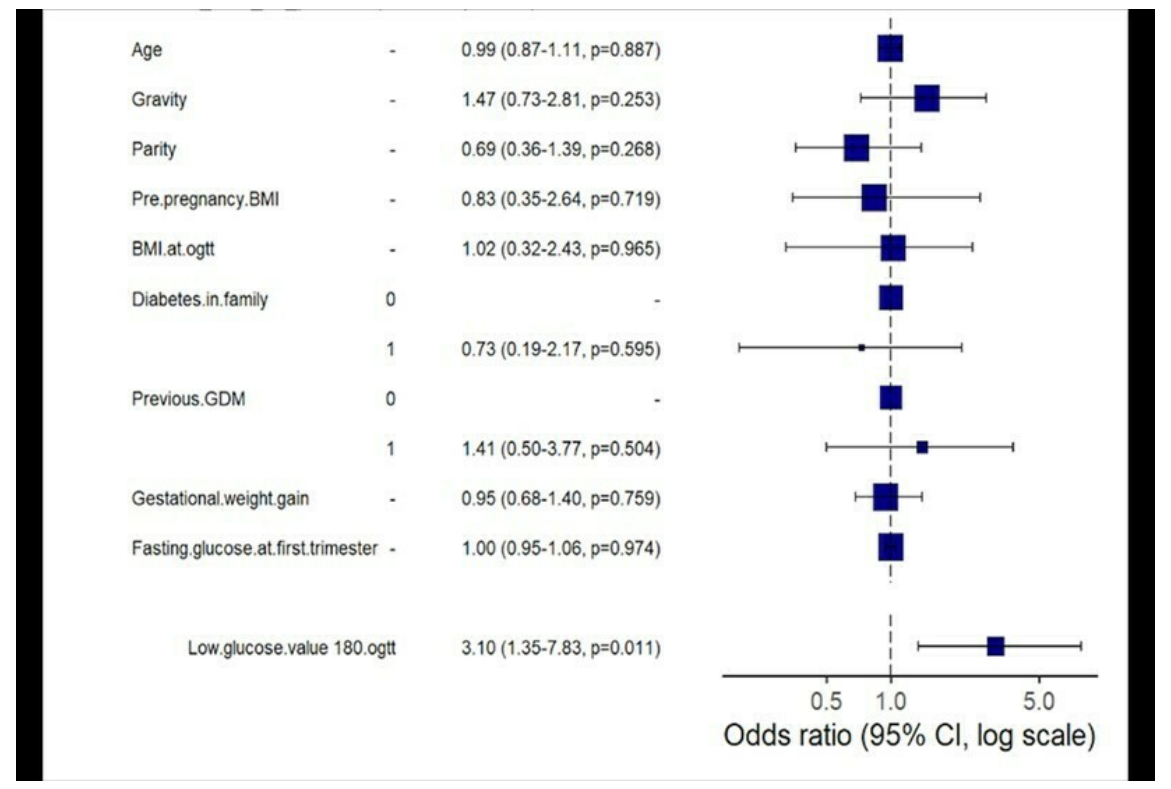

